\title{
A Proposal for an Educational System Service to Support Teaching/Learning Process for Logic Programming
}

\author{
Eric R. G. Dantas, Ryan R. \\ de Azevedo \\ Federal University of \\ Pernambuco-UFPE \\ \{ergd,rra2\}@cin.ufpe.br
}

\author{
Cleyton M. O. Rodrigues, \\ Silas C. Almeida \\ Federal University of \\ Pernambuco - UFPE \\ cmor@cin.ufpe.br, \\ silas.sca@gmail.com
}

\author{
Fred Freitas, Vinicius C. \\ Garcia \\ Federal University of \\ Pernambuco - UFPE \\ $\{$ fred,vcg\}@cin.ufpe.br
}

\begin{abstract}
The Information and Communication Technology (ICT) have been successfully used to transform either partial/total face to face or distance learning education [1]. Educational software systems aid the teaching/learning process, promoting the development of a lot of Virtual Learning Environments (VLEs), improving the assimilation of the content presented in the class. Using mechanisms such as Hardware as a Service (HaaS) and Software as a Service (SaaS), it is possible to envision a new concept: Education as a Service-EdaaS.
\end{abstract}

\section{Proposal}

While the EdaaS becomes available in the cloud, it is possible to expand educational services to the community. It should be noted also that the VLEs do not need to be monitored all the time, since they are cross-platform with higher level of coverage, and further, can be accessed from any host-computing. This results in lower costs of human resources and operational infrastructure. EdaaS, therefore, facilitates and adds more knowledge to students quickly and easily, increasing the level of discussion and interaction between students and teachers in the classroom. In addition, EdaaS may decrease the need for additional computing resources at institutions financially disadvantaged.

In order to demonstrate the potential of EdaaS, we propose the development of an educational service-based platform to improve the teaching/learning process of logic programming. The architecture runs on top of the cloud computing platform, integrating the advantages of this environment, requirements such as usability, flexibility, mobility, extensibility, among others [2]. Similarly to Greenfoot [3] and Scratch [4], the environment is being proposed with characteristics inherent to youth and adults, with more accurate and technical vocabulary, with little use of visuals resources. To evaluate its usefulness, the proposal will be used in experimental classes in beginner courses in Computer Science and Information Systems, in two federal public institutions in Brazil. The environment proposes to use the latest ICTs to deliver education as a service to those who need it, helping to advance in science and education.

\section{References}

[1] Robert Fogel. "The Education Cloud: Delivering Education as a Service”. Whitepaper. 2010. In: http://www.intel.com/assets/pdf/whitepaper/delivering-education-as-a-service.pdf. Access: 12/2010;

[2] M. Armbrust, A. Fox, R. Griffith, et al. "A View of Cloud Computing". Magazine Communications of the ACM CACM Homepage archive. Volume 53, Issue 4, April 2010. ACM New York, USA;

[3] M. Kölling. "Introduction to Programming with Greenfoot - Object-Oriented Programming in Java with Games and Simulations". Pearson Education.2009;

[4] Maloney, J., Resnick, M., Rusk, N., Silverman, B., and Eastmond, E. "The scratch programming language and environment”. ACM Trans. Comput. Educ. 10, 4, Article 16 (11/2010), 15 pages. 